\title{
A map from 1d Quantum Field Theory to Quantum Chaos on a 2d Torus
}

\author{
Tomaž Prosen \\ Physics Department, Faculty of Mathematics and Physics, University of Ljubljana, Jadranska 19, 1111 Ljubljana, Slovenia
}

(June 19, 2021)

\begin{abstract}
Dynamics of a class of quantum field models on 1d lattice in Heisenberg picture is mapped into a class of 'quantum chaotic' one-body systems on configurational 2d torus (or 2d lattice) in Schrödinger picture. Continuum field limit of the former corresponds to quasi-classical limit of the latter.
\end{abstract}

PACS numbers: 05.45.+b, 05.30.Fk, 03.65.Fd, 72.10.Bg

Understanding of long-time dynamics of interacting quantum many-body systems or quantized fields is a long standing open problem. In particular, one would like to understand the conditions for the emergence of quantum mixing (implying ergodicity), i.e. general decay of time autocorrelation functions. Inspired by rich quantum behaviour of non-integrable few body systems having (partially) chaotic classical limit [1] few papers appeared recently concerning 'quantum chaos' in nonintegrable many-body systems [2, 4 . In [4] dynamical phase transition from non-ergodic dynamics exhibiting ideal transport to mixing dynamics exhibiting normal transport has been demonstrated numerically in a nonintegrable kicked $\mathrm{t}-\mathrm{V}$ fermion model on $1 \mathrm{~d}$ lattice.

Below we construct an exact linear mapping from dynamics of a certain large class of interacting infinite spin $-\frac{1}{2}$ chains in Heisenberg picture to a class of non-linear One-Body Image dynamical systems (OBI) in Schrödinger picture which are realized either on configurational $2 \mathrm{~d}$ torus or on $2 \mathrm{~d}$ lattice (tight-binding (TB) formulation). Further we will show by working out two examples: (i) how integrable behavior of the infinite $X X$ spin chain in spatially modulated transversal magnetic field is connected to the integrability of OBI and to the Harper equation [5], and (ii) demonstrate the phase transition of the related non-integrable kicked $X X$ chain from non-ergodic dynamics to ergodic and mixing dynamics corresponding to the stochastic transition from regular to chaotic motion of OBI in the classical limit.

Let $\sigma_{j}^{s}, j \in \mathbb{Z}, s \in\{x, y, z\}$ denote a chain of independent Pauli spin $-\frac{1}{2}$ variables. We start by generalizing the result of [6], namely we find that the operator space spanned by the following set of spatially modulated observables $\left\{U_{n}(\vartheta), V_{n}(\vartheta) ; n \in \mathbb{Z}, \vartheta \in[-\pi, \pi)\right\}$

$$
\begin{aligned}
& U_{n}(\vartheta)=\sum_{j=-\infty}^{\infty} e^{i\left(j+\frac{1}{2}|n|\right) \vartheta} \begin{cases}\sigma_{j}^{x}\left(\sigma_{j}^{z}\right)_{n-1} \sigma_{j+n}^{x} & n \geq 1, \\
-\sigma_{j}^{z} & n=0, \\
\sigma_{j}^{y}\left(\sigma_{j}^{z}\right)_{-n-1} \sigma_{j-n}^{y} & n \leq-1,\end{cases} \\
& V_{n}(\vartheta)=\sum_{j=-\infty}^{\infty} e^{i\left(j+\frac{1}{2}|n|\right) \vartheta} \begin{cases}\sigma_{j}^{x}\left(\sigma_{j}^{z}\right)_{n-1} \sigma_{j+n}^{y} & n \geq 1, \\
1 & n=0, \\
-\sigma_{j}^{y}\left(\sigma_{j}^{z}\right)_{-n-1} \sigma_{j-n}^{x} & n \leq-1 .\end{cases}
\end{aligned}
$$

where $\left(\sigma_{j}^{z}\right)_{k}:=\prod_{l=1}^{k} \sigma_{j+l}^{z}$ for $k \geq 1$ and $\left(\sigma_{j}^{z}\right)_{0}:=1$, is closed under the Lie bracket $[A, B]=A B-B A$ and forms an infinitely dimensional Dynamical Lie Algebra (DLA)

$$
\begin{aligned}
& {\left[U_{n}(\vartheta), U_{l}(\varphi)\right] }=2 i \exp \left(\frac{i}{2}(l \vartheta+n \varphi) s_{l-n}\right) V_{n-l}(\vartheta+\varphi) \\
&-2 i \exp \left(\frac{i}{2}(l \vartheta+n \varphi) s_{n-l}\right) V_{l-n}(\vartheta+\varphi), \\
& {\left[U_{n}(\vartheta), V_{l}(\varphi)\right] }=2 i \exp \left(\frac{i}{2}(-l \vartheta+n \varphi) s_{l}\right) U_{n+l}(\vartheta+\varphi) \\
&-2 i \exp \left(\frac{i}{2}(l \vartheta+n \varphi) s_{l}\right) U_{n-l}(\vartheta+\varphi), \\
& {\left[V_{n}(\vartheta), V_{l}(\varphi)\right]=}\left(s_{n}+s_{l}\right)\left\{\sin \left(\frac{1}{2}(l \vartheta+n \varphi)\right) \times\right. \\
&\left(\left(s_{n-l} s_{l}+1\right) V_{n-l}(\vartheta+\varphi)-\left(s_{l-n} s_{l}+1\right) V_{l-n}(\vartheta+\varphi)\right) \\
&\left.+2 \sin \left(\frac{1}{2}(l \vartheta-n \varphi)\right) V_{n+l}(\vartheta+\varphi)\right\} .
\end{aligned}
$$

where $s_{n}:=-1,0,1$ for $n<,=,>0$, resp., is a sign of integer $n$. Few notable members of DLA are: Ising or XX hamiltonian $H_{I}=J U_{1}(0), H_{X X}=J\left(U_{1}(0)+U_{-1}(0)\right)$, spin interaction with modulated transversal magnetic field $h_{z}=h \cos (\epsilon j)$ with period $2 \pi / \epsilon$ lattice spacings $H_{m h}=\frac{1}{2} h\left(U_{0}(\epsilon)+U_{0}(-\epsilon)\right)$, spin current $j_{s}=V_{1}(0)+$ $V_{-1}(0)$, etc. Let us fix the fundamental field modulation $\epsilon$ and introduce the following notation:

$$
\begin{aligned}
(n, k) \in \mathbb{Z}^{2}, \quad U_{n, k}^{ \pm} & =\frac{1}{2}\left(U_{n}(k \epsilon) \pm U_{-n}(-k \epsilon)\right), \\
V_{n, k}^{+} & =\frac{1}{2}\left(V_{n}(k \epsilon)+V_{-n}(-k \epsilon)\right) s_{n}, \\
V_{n, k}^{-} & =\frac{1}{2}\left(V_{n}(k \epsilon)-V_{-n}(-k \epsilon)\right), \\
W_{n, k}^{ \pm} & =U_{n, k}^{ \pm}+i V_{n, k}^{ \pm}, \\
(y, x) \in \mathbb{T}^{2}, \quad W^{ \pm}(y, x) & =\frac{1}{2 \pi} \sum_{n, k=-\infty}^{\infty} e^{i(n y+k x)} W_{n, k}^{ \pm} .
\end{aligned}
$$

We may also consider $\epsilon$ as a lattice spacing and treat $W_{n, k}^{ \pm}$as a set of spatially $2 \pi$-periodic fields. We will assume that the modulation is incommensurable with the lattice spacing, i.e. that $\epsilon / 2 \pi$ is irrational, otherwise obsrvables $W_{n, k}^{ \pm}$are periodic w.r.t. index $k$. DLA becomes a Hilbert space when we introduce an infinite temperature (grand) canonical scalar product [6] $(A \mid B):=\lim _{L \rightarrow \infty} \frac{1}{L} 2^{-L} \operatorname{tr} A^{\dagger} B$, where $L$ is a diverging length of the spin-chain. Let the two linear subspaces spanned by $W_{n, k}^{\sigma}\left(\right.$ or $\left.W^{\sigma}(y, x)\right)$ for $\sigma \in\{+,-\}$ be denoted by $\mathfrak{M}_{\sigma}$. The spaces $\mathfrak{M}_{+}$and $\mathfrak{M}_{-}$are orthogonal and observables $W_{n, k}^{+}(n, k \in \mathbb{Z})$ and $W_{n, k}^{-}($for $n \geq 1)$ form orthonormal bases in each of them, since one can show

$$
\begin{aligned}
& \left(W_{n, k}^{+} \mid W_{m, l}^{+}\right)=\delta_{n, m} \delta_{k, l}, \quad\left(W_{n, k}^{+} \mid W_{m, l}^{-}\right)=0, \\
& \left(W_{n, k}^{-} \mid W_{m, l}^{-}\right)=\left(\delta_{n, m}-\delta_{n,-m}\right) \delta_{k, l} .
\end{aligned}
$$


The full set $\left\{W_{n, k}^{-}\right\}$is over-complete, since $W_{-n, k}^{-}=$ $-W_{n, k}^{-}$, while the subspace $\mathfrak{M}_{+}=\mathfrak{M}_{+}^{\dagger}$ is self-adjoint, since $W_{n, k}^{+\dagger}=W_{-n,-k}^{+}$. One can write analogous relations in terms of continuous variables $(y, x)$. We have $\mathrm{DLA}=\mathfrak{M}_{-}^{\dagger} \oplus \mathfrak{M}_{+} \oplus \mathfrak{M}_{-}$. Note that the adjoint map $(\operatorname{ad} A) B=[A, B]$ generates the Heisenberg motion on DLA, $\exp (i t \operatorname{tad} A) B=e^{i t A} B e^{-i t A}$. In particular, the motion generated by $U_{n, k}^{+}$has a beautiful structure. Let us write the self-adjoint Hamiltonian in a general form as

$$
H=\sum_{n, k} \frac{1}{4} g_{n, k}\left(U_{n, k}^{+} e^{i \gamma_{n, k}}+U_{n,-k}^{+} e^{-i \gamma_{n,-k}}\right)
$$

using two sets of possibly time dependent real coefficients $g_{n, k}=g_{n, k}(t), \gamma_{n, k}=\gamma_{n, k}(t)$. Tedious but straightforward calculation, using algebra (1i), gives the action of ad $H$ on two continuous sets of observables $W^{+}(y, x), W^{-}(y, x),(y, x) \in \mathbb{T}^{2}$ which can be written in terms of two non-local 'Schrödinger operators' $\hat{\mathcal{H}}^{ \pm}$

$$
\begin{gathered}
(\operatorname{ad} H) W^{ \pm}(y, x)=-\frac{1}{\hbar} \hat{\mathcal{H}}^{ \pm} W^{ \pm}(y, x), \\
\hat{\mathcal{H}}^{+}=\sum_{n, k} \hbar g_{n, k}\left(\sin \left(n \hat{p}_{x}-k \hat{p}_{y}\right) \sin \left(k x+n y-\gamma_{n, k}\right)\right. \\
\left.-\sin \left(n \hat{p}_{x}+k \hat{p}_{y}\right) \sin \left(k x-n y-\gamma_{n, k}\right)\right), \\
\hat{\mathcal{H}}^{-}=\sum_{n, k} \hbar g_{n, k}\left(\cos \left(n \hat{p}_{x}-k \hat{p}_{y}\right) \cos \left(k x+n y-\gamma_{n, k}\right)\right. \\
\left.+\cos \left(n \hat{p}_{x}+k \hat{p}_{y}\right) \cos \left(k x-n y-\gamma_{n, k}\right)\right),
\end{gathered}
$$

where $\hat{p}_{x, y}=-i \hbar \partial / \partial_{x, y}$ are momentum operators conjugate to $x, y$ with an 'effective Planck constant' [7]

$$
\hbar=\frac{1}{2} \epsilon
$$

Since Heisenberg dynamics generated by $H$ is closed on $\mathfrak{M}_{\sigma},(\operatorname{ad} H) \mathfrak{M}_{\sigma} \subseteq \mathfrak{M}_{\sigma}$, one may write a general timeevolving operator $A(t) \in \mathfrak{M}_{\sigma}$ in terms of a complexvalued 'Schrödinger wave function', in either 'momentum' $\Psi_{n, k}^{A}(t)$ or 'position' $\Psi^{A}(y, x ; t)$ representation

$$
A(t)=\sum_{n, k} \Psi_{n, k}^{A}(t)^{*} W_{n, k}^{\sigma}=\int_{\mathbb{T}^{2}} d y d x \Psi^{A}(y, x ; t)^{*} W^{\sigma}(y, x) .
$$

By means of eq. (雨) and the fact that $\hat{\mathcal{H}}^{\sigma}$ is Hermitian on $L^{2}\left(\mathbb{T}^{2}\right)$ (which can be checked directly using the expressions (5)) one can easily show that the Heisenberg evolution of the observable $A(t),(d / d t) A(t)=i(\operatorname{ad} H) A$, is fully equivalent to the Scrödinger equation

$$
i \hbar \frac{d}{d t} \Psi^{A}(y, x ; t)=\hat{\mathcal{H}}^{\sigma} \Psi^{A}(y, x ; t) .
$$

governing time evolution of one particle on a torus $\mathbb{T}^{2}$ (OBI). The bilinear map $(H, A(t)) \leftrightarrow\left(\hat{\mathcal{H}}^{\sigma}, \Psi^{A}(y, x ; t)\right)$ is a central result of this Letter. To conclude a general exposition we make few remarks: (i) A non-trivial 'classical limit' $\hbar \rightarrow 0$ of OBI exists, being equivalent (6) to the continuum field limit of the quantum spin chain model $\epsilon \rightarrow 0$, if $\hbar g_{n, l}$ (and not $g_{n, l}$ alone) are kept constant and finite. (ii) The operators $\hat{\mathcal{H}}^{+}$and $\hat{\mathcal{H}}^{-}$(5) commute

$$
\left[\hat{\mathcal{H}}^{+}, \hat{\mathcal{H}}^{-}\right] \equiv 0,
$$

and the Poisson bracket of the corresponding classical counterparts vanishes. (iii) As a consequence of the previous remark we find that OBI (7) (and its classical limit) is integrable, $\hat{\mathcal{H}}^{-\sigma}$ being the second integral of motion, provided the original spin-field Hamiltonian $H$ (3) or OBI Hamiltonian $\hat{\mathcal{H}}^{\sigma}$ is autonomous, i.e. $(\partial / \partial t) H \equiv 0$. However, one has a possibility of chaotic motion in classical limit and emergence of 'quantum chaos' when the problem is explicitly time-dependent, say that coefficients are periodic functions, $g_{n, k}(t+1)=g_{n, k}(t)$. In such case one integrates the evolution over one period of time and defines the unitary Floquet maps $U=\hat{\mathcal{T}} \exp \left(-i \int_{0}^{1} d t H(t)\right)$, $\hat{\mathcal{U}}^{\sigma}=\hat{\mathcal{T}} \exp \left(-i \int_{0}^{1} d t \hat{\mathcal{H}}^{\sigma}(t)\right)$. (iv) Temporal correlation functions of the quantum field problem are mapped (using eqs. (2)) onto transition amplitudes of OBI

$$
\left(A(t) \mid B\left(t^{\prime}\right)\right)= \begin{cases}\left\langle\Psi^{A}(t) \mid \Psi^{B}\left(t^{\prime}\right)\right\rangle & \sigma=+, \\ \left\langle\Psi^{A}(t) \mid \hat{\mathcal{P}}_{y} \Psi^{B}\left(t^{\prime}\right)\right\rangle & \sigma=-,\end{cases}
$$

where $\hat{\mathcal{P}}_{y} \Psi(y, x)=\Psi(y, x)-\Psi(-y, x)$. Therefore, ergodic properties of many-body dynamics on DLA are determined by the spectral properties of OBI: (a) Spin chain is quantum mixing in $\mathfrak{M}_{\sigma}, \lim _{t \rightarrow \infty}(A(t) \mid B)=$ $0, A, B \in \mathfrak{M}_{\sigma}$, iff the spectrum of OBI Hamiltonian $\hat{\mathcal{H}}^{\sigma}$ (or of OBI Floquet map $\hat{\mathcal{U}}^{\sigma}$ ) does not have (nontrivial) point component. (b) Spin chain is quantum ergodic in $\mathfrak{M}_{\sigma}, \lim _{T \rightarrow \infty} T^{-1} \int_{0}^{T} d t(A(t) \mid B)=0$, iff 0 (or 1 ) is not in the non-trivial point spectrum of $\hat{\mathcal{H}}^{\sigma}$ (or $\left.U^{\sigma}\right)$. In autonomous case, $\partial H / \partial t \equiv 0$, the Hamiltonian $H$ and the trivial zero-frequency eigenstate, $\hat{\mathcal{H}}^{+} \Psi^{H}=$ $-\hbar \Psi^{[H, H]}=0, \Psi_{n, k}^{H}=\frac{1}{8}\left(g_{n, k} e^{-i \gamma_{n, k}}+g_{n,-k} e^{i \gamma_{n,-k}}+\right.$ $\left.g_{-n, k} e^{-i \gamma_{-n, k}}+g_{-n,-k} e^{i \gamma_{-n,-k}}\right)$ should be excluded from $\mathfrak{M}_{+}$and $L^{2}\left(\mathbb{T}^{2}\right)$, respectively, i.e. $(A \mid H)=(B \mid H)=0$.

We apply the above results to work out two interesting examples. Example I: XX spin chain in spatially modulated quasi-periodic transversal magnetic field $\vec{h}_{j}=(0,0, h \cos (\epsilon j))(\mathrm{XXmh})$

$$
H=H_{X X}+H_{m h}=J U_{1,0}^{+}+\frac{1}{2} h\left(U_{0,1}^{+}+U_{0,-1}^{+}\right) .
$$

Here the Heisenberg dynamics on DLA is governed by the following commuting one-body problems

$$
\begin{aligned}
& \hat{\mathcal{H}}^{+}=\alpha \sin \hat{p}_{x} \sin y-\beta \sin \hat{p}_{y} \sin x, \\
& \hat{\mathcal{H}}^{-}=\alpha \cos \hat{p}_{x} \cos y+\beta \cos \hat{p}_{y} \cos x,
\end{aligned}
$$

where $\alpha=2 \epsilon J=4 \hbar J, \beta=2 \epsilon h=4 \hbar h$. This models are directly related to the electron motion on $2 \mathrm{~d}$ rectangular $a \times b$ lattice in a uniform perpendicular magnetic field $h^{\prime}$ within the TB approximation [5,8]. In the symmetric gauge $\vec{A}=\frac{1}{2} h^{\prime}(-y, x, 0)$ the TB problem with the band energy $\mathcal{E}(\vec{K})=\frac{\alpha}{2} \cos \left(a K_{1}\right)+\frac{\beta}{2} \cos \left(b K_{2}\right)$ reads 


$$
\begin{aligned}
\hat{\mathcal{H}} \Psi_{n, k} & =\frac{\alpha}{2}\left(e^{i \frac{1}{2} \epsilon k} \Psi_{n+1, k}+e^{-i \frac{1}{2} \epsilon k} \Psi_{n-1, k}\right) \\
& +\frac{\beta}{2}\left(e^{-i \frac{1}{2} \epsilon n} \Psi_{n, k+1}+e^{i \frac{1}{2} \epsilon n} \Psi_{n, k-1}\right)
\end{aligned}
$$

where $\epsilon=e_{o} a b h^{\prime} / c_{o} \hbar_{\text {phys }}$ [7] is here the dimensionless magnetic flux thru lattice cell. We note that discrete indices $(n, k) \in \mathbb{Z}^{2}$ now label the position lattice $(n a, k b)$ while continuous indices $(y, x) \in \mathbb{T}^{2}$ are the conjugate quasi-momenta. OBI Hamiltonians $\hat{\mathcal{H}}^{ \pm}$can be written in terms of $\hat{\mathcal{H}}$ and its time-reversal $\hat{\mathcal{H}}^{*}=\left.\hat{\mathcal{H}}\right|_{h^{\prime} \rightarrow-h^{\prime}}$, namely $\hat{\mathcal{H}}^{ \pm}=\hat{\mathcal{H}} \mp \hat{\mathcal{H}}^{*}$, and hence $\left[\hat{\mathcal{H}}, \hat{\mathcal{H}}^{*}\right]=0$. Using a different, Landau gauge $\vec{A}=h^{\prime}(0, x, 0)$ the TB problem (12) can be re-written in terms of $1 \mathrm{~d}$ Harper equation [5]

$$
\frac{1}{2} \alpha\left(u_{n+1}+u_{n-1}\right)+\beta \cos (n \epsilon-\vartheta) u_{n}=E u_{n} .
$$

Let us assume for the moment that $\alpha<\beta$. Then $u_{n}(\vartheta ; E)=u_{n}$ is a unique exponentially localized eigenfunction (EF) of eq. (13) which has a dense pure point spectrum, and $\Psi_{n, k}(\vartheta ; E)=\exp \left(i\left(\vartheta-\frac{1}{2} \epsilon n\right) k\right) u_{n}(\vartheta ; E)$ is a degenerate dense set of EFs of TB problem (12), $\hat{\mathcal{H}} \Psi_{n, k}(\vartheta ; E)=E \Psi_{n, k}(\vartheta ; E)$, for a dense set of parameters $\vartheta$ [8]. Though $\hat{\mathcal{H}}$ and $\hat{\mathcal{H}}^{*}$ should have a common set of EFs, $\Psi_{n, k}(\vartheta ; E)$ is not an EF of $\hat{\mathcal{H}}^{*}$, neither it is in $L^{2}$ since it is extended in variable $k$. We search for such $\mathrm{EF}$ with an ansatz $\Phi_{n, k}\left(\vartheta ; E, E^{\prime}\right)=\sum_{j} v_{j} \Psi_{n, k}(\vartheta+\epsilon j ; E)$ and require $\hat{\mathcal{H}}^{*} \Phi_{n, k}\left(\vartheta ; E, E^{\prime}\right)=E^{\prime} \Phi_{n, k}\left(\vartheta ; E, E^{\prime}\right)$ yielding the Harper equation (13) for coefficients $v_{n}=u_{n}\left(\vartheta ; E^{\prime}\right)$. Thus we obtain a common set of EFs of $\hat{\mathcal{H}}$ and $\hat{\mathcal{H}}^{*}$ in terms of a 'convolution' of two 1d Harper functions

$\Phi_{n, k}\left(\vartheta ; E, E^{\prime}\right)=\sum_{l} u_{l+n}(\vartheta ; E) u_{l}\left(\vartheta ; E^{\prime}\right) e^{i\left(\vartheta-\epsilon l-\frac{1}{2} \epsilon n\right) k}$

which is also a common set of EFs of $\hat{\mathcal{H}}^{ \pm}(11)$

$$
\hat{\mathcal{H}}^{ \pm} \Phi_{n, k}\left(\vartheta ; E, E^{\prime}\right)=\left(E \mp E^{\prime}\right) \Phi_{n, k}\left(\vartheta ; E, E^{\prime}\right) .
$$

The property $\Phi_{n, k}\left(\vartheta+\epsilon ; E, E^{\prime}\right)=\Phi_{n, k}\left(\vartheta ; E, E^{\prime}\right)$ suggests independence of EF on parameter $\vartheta$ provided $\epsilon / 2 \pi$ is irrational. If $\alpha>\beta$ localized EFs can be constructed analoglously by 'duality transformation' $n \leftrightarrow k, y \leftrightarrow x$. Thus, we found that OBI $\hat{\mathcal{H}}^{ \pm}$have a dense pure point spectrum for $\alpha \neq \beta$, hence XXmh is non-mixing, nonergodic and even completely integrable, namely 'zeroenergy' eigenstates of $\hat{\mathcal{H}}^{\sigma}$ are the images of a complete set of conserved charges, $Q_{\sigma}(E)=\sum_{n, k} \Phi_{n, k}^{*}(E, \sigma E) W_{n, k}^{\sigma}$, $\left[H, Q_{\sigma}(E)\right] \equiv 0$. However, time autocorrelation function $(A(t) \mid A)$ of a certain observable $A$ may still decay to zero provided the image function $\Psi^{A}$ is orthogonal to all (localized) EFs (14) of $\hat{\mathcal{H}}^{ \pm}$. Interestingly, this happens with the spin-current $j_{s}=W_{1,0}^{+}-$ $W_{-1,0}^{+}$if $\alpha<\beta$ (i.e. $J<h$ ), since EFs (14) have the following properties $\Phi_{n, k}\left(E, E^{\prime}\right)^{*}=\Phi_{n, k}\left(E^{\prime}, E\right)=$ $\Phi_{-n,-k}\left(E, E^{\prime}\right)$ and $\Phi_{n, 0}\left(E, E^{\prime}\right)^{*}=\Phi_{n, 0}\left(E, E^{\prime}\right)$ (we put $\vartheta:=0)$ implying $\Phi_{n, 0}\left(E, E^{\prime}\right)=\Phi_{-n, 0}\left(E, E^{\prime}\right)$. So we have $\left\langle\Psi^{j_{s}} \mid \Phi\left(E, E^{\prime}\right)\right\rangle \equiv 0$. This proves $\left(j_{s}(t \rightarrow \infty) \mid j_{s}\right) \rightarrow 0$ and non-ballistic spin-transport (vanishing spin stiffness $\left.D_{s}:=\lim _{T \rightarrow \infty}(1 / T) \int_{0}^{T} d t\left(j_{s}(t) \mid j_{s}\right)\right)$ for $J<h$, while for $J>h$ we find in general ballistic transport $\left(D_{s}>0\right)$ since no similar symmetry exists for the other index $k$.

Example II: kicked XXmh model (kXXmh) with timedependent Hamiltonian

$$
H(t)=J U_{1,0}^{+}+\frac{1}{2} h\left(U_{0,1}^{+}+U_{0,-1}^{+}\right) \sum_{m} \delta(t-m) .
$$

One-period propagator from just after the kick

$$
U=\exp \left(-i \frac{1}{2} h\left(U_{0,1}^{+}+U_{0,-1}^{+}\right)\right) \exp \left(-i J U_{1,0}^{+}\right)
$$

is equivalent to Floquet quantum maps of two kicked OBI

$$
\begin{aligned}
& \hat{\mathcal{U}}^{+}=\exp \left(\frac{i \beta}{\hbar} \sin \hat{p}_{y} \sin x\right) \exp \left(\frac{-i \alpha}{\hbar} \sin \hat{p}_{x} \sin y\right) \\
& \hat{\mathcal{U}}^{-}=\exp \left(\frac{-i \beta}{\hbar} \cos \hat{p}_{y} \cos x\right) \exp \left(\frac{-i \alpha}{\hbar} \cos \hat{p}_{x} \cos y\right) .
\end{aligned}
$$

In the following we will consider only the map $\hat{\mathcal{U}}^{+}$since the space $\mathfrak{M}_{+}$contain physically more interesting observables. The Floquet evolution $\Psi^{A}(m)=\hat{\mathcal{U}}^{+m} \Psi^{A}(0)$ yielding Heisenberg evolution of observables $A(m) \in \mathfrak{M}_{+}$is in the 'classical' limit equivalent to a volume-preserving $(2 \times 2)$ d map on $\mathbb{T}^{2} \times \mathbb{R}^{2}$

$$
\begin{aligned}
& x^{\prime}=x+\alpha \cos p_{x} \sin y, \quad p_{y}^{\prime}=p_{y}-\alpha \sin p_{x} \cos y, \\
& y^{\prime}=y-\beta \cos p_{y}^{\prime} \sin x^{\prime}, \quad p_{x}^{\prime}=p_{x}+\beta \sin p_{y}^{\prime} \cos x^{\prime},
\end{aligned}
$$

which is non-integrable and (almost) fully chaotic for sufficiently large kick parameters, $\alpha, \beta \gg 1$. Interesting question is now if and when the dynamics of $\mathrm{kXXmh}$ is quantum mixing and how it corresponds to dynamics of the 'classical map' (19) as $\hbar=\frac{1}{2} \epsilon \rightarrow 0$. This problem has been approached numerically by iterating the one-body Floquet map $\hat{\mathcal{U}}^{+}$on a finite (truncated) momentum space $(n, k) \in\{-N / 2 \ldots N / 2\}^{2}$. The position states are then discretized as $x_{j}=s j, y_{j}=s j, s=2 \pi / N$. The truncated Floquet map $\hat{\mathcal{U}}^{+}$can be efficiently implemented by means of Fast Fourier Transformation (FFT), namely if $F$ is $1 \mathrm{~d}$ FFT on $N$ sites then $N^{2} \times N^{2}$ Floquet matrix is de-composed as

$\left(F^{-1} \otimes 1\right)\left(\operatorname{diag} C_{n, k}\right)\left(F \otimes F^{-1}\right)\left(\operatorname{diag} D_{n, k}\right)(1 \otimes F)$, with diagonal matrices $C_{n, k}=\exp (i(\beta / \hbar) \sin s n \sin \hbar k)$ and $D_{n, k}=\exp (-i(\alpha / \hbar) \sin \hbar n \sin s k)$, requiring $\sim$ $4 N^{2} \log _{2} N$ computer operations per time step. In order to avoid recurrences of quantum probability due to finiteness of momentum space we use an absorbing boundary in momentum space, namely after each iteration of the truncated Floquet map we multiply the wave-function by a box-window, $\Psi_{n, k}(m) \rightarrow \theta(N / 2-\alpha / \hbar-|n|) \theta(N / 2-$ $\beta / \hbar-|k|) \Psi_{n, k}(m)$, Convergence to true dynamics on a torus has been checked by comparing results for different truncations, say $N$ and $N / 2$ (we went up to $N=2^{14}$ ).

In Fig.1 we show numerical results for the autocorrelation function of the spin current $C(m)=$ $\left(j_{s}(m) \mid j_{s}(0)\right)$ while similar, compatible results have been 
obtained for the time-correlations of other observables. (i) For sufficiently large kick parameters $\alpha, \beta$ the classical map (19) is strongly chaotic and mixing exhibiting normal diffusion in momentum plane $\left(p_{x}, p_{y}\right)$. However, $\mathrm{kXXmh}$ is not exactly mixing for any finite $\hbar:|C(m)|$ is rapidly (possibly exponentially) decreasing down to some value $C^{*}=\overline{|C(m)|}$ where it saturates. When we decrease $\hbar, C^{*}$ decreases proportionally, $C^{*} \propto \hbar$, and so in the 'quasi-classical'/continuum limit $\hbar=\frac{1}{2} \epsilon \rightarrow 0$ the point spectrum of $\hat{\mathcal{U}}^{+}$vanishes and kXXmh approaches mixing behaviour in accordance with the map (19). (ii) For smaller but still finite values of $\alpha, \beta$ the classical map enters into the regime of KAM quasi-integrability with invariant tori suppressing the diffusion of momenta $\left(p_{x}, p_{y}\right)$. Correspondingly, $\mathrm{kXXmh}$ is non-mixing and $C^{*} \sim 1$ for any value of $\hbar$. In this regime, $C(m)$ is very weakly $\hbar$-dependent. In both regimes, (i) and (ii), the square widths of the 'wavepackets' $\left\langle\Psi^{j_{s}}(m)\left|\hat{p}_{x, y}^{2}\right| \Psi^{j_{s}}(m)\right\rangle$ have been found to be uniformly increasing in time and limited only by the size of the truncated momentum space $N$. This rules out the possibility of quantum localization and existence of pure point spectrum, and indicates coexistence of point and continuous spectrum for any finite $\hbar$ (and finite $\alpha, \beta$ ), a situation similar to (possibly related) $1 \mathrm{~d}$ kicked Harper model [9]. In the limit $\alpha, \beta \rightarrow 0$, the continuous spectral component vanishes and we recover integrable XXmh model with pure point spectrum as discussed above. The quantum correlation function $C(m)$ seem to follow the quasi-classical propagator only up to logarithmically short time, namely we found empirically that deviation (when it is small) increases exponentially $\left|C(m)-C_{\hbar \rightarrow 0}(m)\right| \approx 0.022 \hbar^{2} e^{\lambda m}$ with $\lambda \approx 0.59$ for $\alpha=3, \beta=0.75$ and $\lambda \approx 1.1$ for $\alpha=6, \beta=1.5$.

Conclusions. In a specific $\infty$ d class of (Pauli spin, or spinless fermion) quantum field models in $1 \mathrm{~d}$, the Heisenberg time evolution in two disjoint $\infty \mathrm{d}$ linear subspaces of essential field observables has been shown to be formally equivalent to the Schrödinger dynamics of a class of one-body image problems on a $2 \mathrm{~d}$ torus (or $2 \mathrm{~d}$ lattice). Autonomous models of this class were found to be completely integrable, pointing out a novel class of integrable one-body problems (5,8). For example, dynamics of XX chain in a static quasiperiodic transversal field has been solved in terms of Harper equation [10]. However, time-dependent (e.g. periodically kicked) models of our class behave in a non-integrable fashion being mapped onto one-body problems with chaotic classical limit. It seems that spatial modulation is crucial to break integrability since spin chain kicked with homogeneous transversal field remains completely integrable as found in [6]. In the contunuum field limit our kicked spin chain model (kXXmh) has been demonstrated to undergo a (phase) transition from mixing to non-mixing dynamics (similar to a transition found in (何), as its one-body counterpart in the classical limit undergoes a stochastic transi- tion from chaotic to quasi-regular motion. This is an interesting link between quantum field theory and chaotic dynamics and should inspire future research in this direction. Such approach to long-time dynamics of certain (non-integrable) quantum many-body systems, since it makes time evolution formally equivalent to 'quantum chaos' in few degrees of freedom, overcomes the traditional problems due to huge Fock space in thermodynamic limit. Financial support by the Ministry of Science and Technology of R. Slovenia is acknowledged.

[1] G. Casati and B.V. Chirikov, eds., 'Quantum Chaos: Between Order and Disorder', (Cambridge U.P. 1994).

[2] G.J.-Lasinio, C.Presilla, Phys.Rev.Lett.77, 4322 (1996).

[3] G. Montambaux, D. Poilblanc, J. Bellisard, C. Sire, Phys.Rev.Lett.70, 497 (1993); D. Poilblanc, T. Ziman, J. Bellisard, F. Mila, G. Montanbaux, EPL 22, 537 (1993).

[4] T. Prosen, Phys.Rev.Lett. 80 (1998) 1808; J.Phys.A: Math.Gen.31 (1998) L645; e-print cond-mat/9808150.

[5] P.G. Harper, Proc.Phys.Soc.A 68, 874 (1955).

[6] T. Prosen, J. Phys. A: Math. Gen. 31, L397 (1998).

[7] $\hbar$ is a dimensionless model parameter which has to be distinguished from the physical Planck constant $\hbar_{\text {phys }}$.

[8] J.B. Sokoloff, Phys.Rep. 126, 189 (1985).

[9] R. Artuso, G. Casati, F. Borgonovi, L. Rebuzzini, I Guarneri, Int.J.Mod.Phys.B 8, 207 (1994); F. Borgonovi, D. Shepelyansky, EPL 29, 117 (1995).

[10] Harper spectrum of quasi-particle excitations of XXmh was found in I.I.Satija, Phys.Rev.B 49, 3391 (1994).

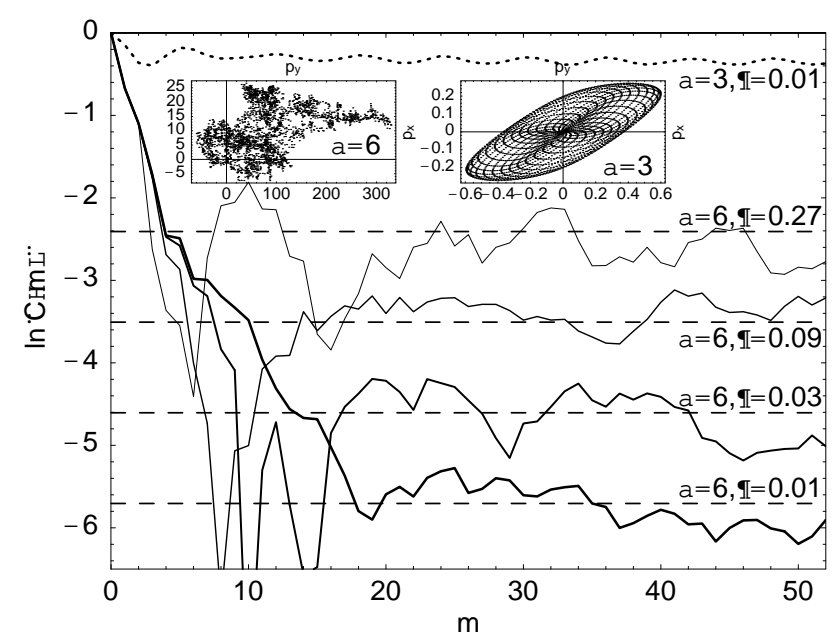

FIG. 1. $\ln |C(m)|$ in $\mathrm{kXXmh}$ for several indicated values of parameters $\alpha$ and $\epsilon$ while $\beta=\alpha / 4$. Note the transition to mixing dynamics as $\epsilon$ is decreased (heavy-light full curves) for 'chaotic case' $\alpha=6$, and stable non-mixing behaviour for 'quasi-regular' case $\alpha=3$ (dotted curve, curves for other (small) values of $\epsilon$ are almost indistinguishable). Broken lines at $\ln |\epsilon / 3|$ indicate the scaling $C^{*} \propto \hbar=\frac{1}{2} \epsilon$. In two insets we show two orbits in momentum plane (chaotic, diffusive for $\alpha=6$ and quasi-regular for $\alpha=3$ ) of the map (19) of length 3000 starting at $x_{0}=0.2, y_{0}=0, p_{x 0}=0, p_{y 0}=0.005$. 